\title{
Distribution of Nucleosides in Populations of Cordyceps cicadae
}

\author{
Wen-Bo Zeng ${ }^{1}$, Hong Yu ${ }^{1}{ }^{1} *$, Feng Ge ${ }^{2}$, Jun-Yuan Yang ${ }^{1}$, Zi-Hong Chen ${ }^{1}$, Yuan-Bing Wang ${ }^{1}$, \\ Yong-Dong Dai ${ }^{1}$ and Alison Adams ${ }^{3}$
}

1 Yunnan Herbal Laboratory, Institute of Herb Biotic Resources, Yunnan University, Kunming 650091, Yunnan, China; E-Mails: zengwenboherb@163.com (W.-B.Z.); icecream6973@sina.com (J.-Y.Y.); czh78@tom.com (Z.-H.C.); wangyb001@126.com (Y.-B.W.); daidiy555@gmail.com (Y.-D.D.) Faculty of Life Science and Technology, Kunming University of Science and Technology, Kunming 650500, Yunnan, China; E-Mail: gefeng79@aliyun.com

3 Department of Biological Sciences, College of Engineering, Forestry and Natural Science, Northern Arizona University, Flagstaff, AZ 86011-5640, USA; E-Mail: Alison.Adams@nau.edu

* Author to whom correspondence should be addressed; E-Mail: herbfish@163.com or hongyu@ynu.edu.cn; Tel.: +86-137-006-766-33; Fax: +86-871-650-346-55.

Received: 15 January 2014; in revised form: 25 April 2014 / Accepted: 5 May 2014 / Published: 14 May 2014

Abstract: A rapid HPLC method had been developed and used for the simultaneous
determination of 10 nucleosides (uracil, uridine, 2'-deoxyuridine, inosine, guanosine,
thymidine, adenine, adenosine, 2'-deoxyadenosine and cordycepin) in 10 populations of
Cordyceps cicadae, in order to compare four populations of Ophicordyceps sinensis and
one population of Cordyceps militaris. Statistical analysis system (SAS) 8.1 was used to
analyze the nucleoside data. The pattern of nucleoside distribution was analyzed in the sampled
populations of $C$. cicadae, $O$. sinensis and $C$. militaris, using descriptive statistical analysis,
nested analysis and Q cluster analysis. The total amount of the 10 nucleosides in coremium
was $1,463.89-5,678.21 \mu \mathrm{g} / \mathrm{g}$ in 10 populations of $C$. cicadae, $1,369.80-3,941.64 \mu \mathrm{g} / \mathrm{g}$ in
sclerotium. The average contents of the 10 analytes were $4,392.37 \mu \mathrm{g} / \mathrm{g}$ and $3,016.06 \mu \mathrm{g} / \mathrm{g}$ in
coremium and sclerotium, respectively. The coefficient of variation $(C V)$ of nucleosides
ranged from $8.36 \%$ to $112.36 \%$ in coremium of $C$. cicadae, and from $10.77 \%$ to $155.87 \%$
in sclerotium of $C$. cicadae. The $C V$ of the nucleosides was wide within $C$. cicadae
populations. The nested variation analysis by the nine nucleosides' distribution indicated
that about $42.29 \%$ of the nucleoside variability in coremium was attributable to the
differentiation among populations, and the remaining $57.71 \%$ resided in the populations. It 
was also shown that about $28.94 \%$ of the variation in sclerotium was expressed between populations, while most of the variation $(71.06 \%)$ corresponded to the populations.

Keywords: Cordyceps cicadae; nucleosides; distribution

\section{Introduction}

Cordyceps cicadae X. Q. Shing (Figure 1), named "Chan Hua", belongs to the genus Cordyceps (family Clavicipitaceae, Ascomycotina), and its anamorph is Isaria cicadae Miq [1], which is a major parasitic fungus growing on the nymph of Cicada flammata Distant, Platypleura kaempferi Fabricius, Crytotympana pustulata Fabricious [2], Platylomia pieli Kato [3] and Oncotympana maculatieollis Motsch (Figure 2). Cordyceps cicadae has been used as a Traditional Chinese Medicine and food for about 1,500 years in China [2], much longer than Ophicordyceps sinensis (Berk.) G. H. Sung, J. M. Sung $[4,5]$.

Figure 1. Cordyceps cicadae used for this study.

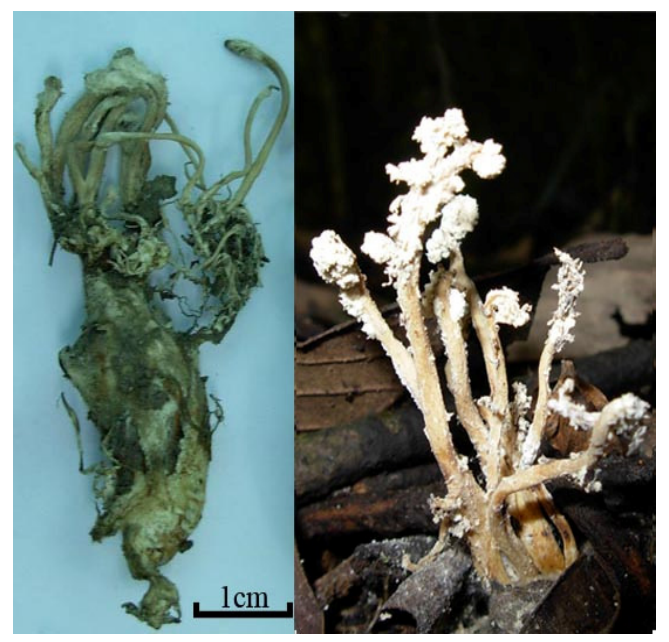

Figure 2. The nymph and adult of Oncotympana maculatieollis, as one host of C. cicadae, collected from Kunming in Yunnan (Pop CCKSG).

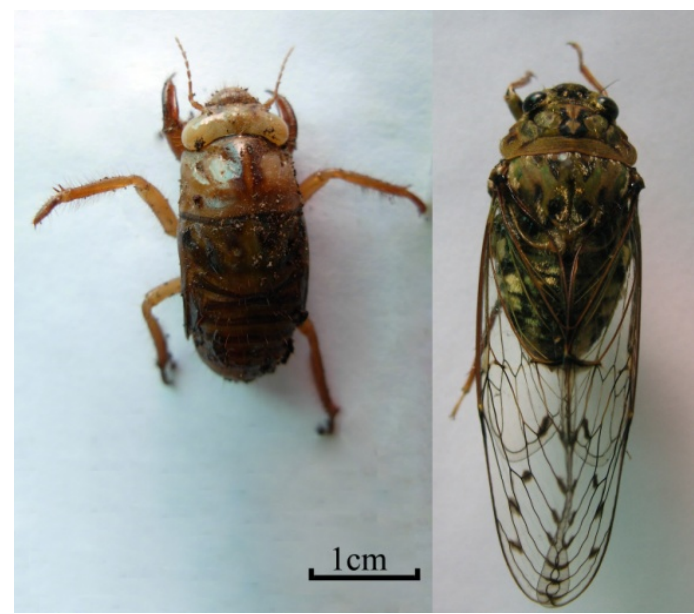


Furthermore, $C$. cicadae has been used as a substitute for $O$. sinensis. Its putative active functions include: (1) treatment of childhood convulsions; (2) antitumor activity [6,7]; (3) analgesic activity and sedative function [3,8]; (4) amelioration of renal function [9]; (5) anti-fatigue effects [10]; (6) immunomodulatory effects [7].

Cordyceps cicadae is a cosmopolitan species in many regions of the World, and its habitat demands are less strict than those of $O$. sinensis. The distribution of $C$. cicadae had been surveyed in China (Table 1). It has also been recorded in South Asia, Europe, North America [11] and Jeju Island in South Korea [12,13].

Table 1. Distribution of C. cicadae in China.

\begin{tabular}{cc}
\hline Province & Location \\
\hline Yunnan & Mojiang, Fengyang [13] Lanping, Weixi, Xianggelila, Zhaotong and Kunming \\
Sichuan & Mount Emei, Qingcheng mountain and Qingyun mountain [13] and Xiangcheng \\
Guizhou & Fanjing mountain, Libo karst geopark, Guiyang forest park and Huaxi [13,14] \\
Jiangsu & Yixing \\
Guangxi & Leye [12] \\
Hainan & Wuzhi mountain [12] \\
Fujian & Wushan in Fuzhou [13] \\
Shanghai & Tianma mountain [15] \\
Zhejiang & Hangzhou [16] \\
Guangdong [11] & $-{ }^{a}$ \\
Hunan [11] & - \\
Hubei [11] & - \\
\hline
\end{tabular}

${ }^{\mathrm{a}}$ No details.

According to Traditional Chinese Medicine, C. cicadae had been considered as a drug similar to $O$. sinensis, with its effective composition of amino acids, polysaccharides, and mannitol being similar to those of $O$. sinensis [17]. Several components, such as nucleosides, polysaccharides, ergosterol and mannitol, had been used as markers for quality control of Cordyceps and its products [4]. The following chemical constituents have been isolated from C. cicadae: polysaccharides [18,19], galactomannan [20], adenosine, uridine, inosine, guanosine [21], ISP-1(myriocin) [22,23] and ergosterol peroxide [24].

Previous studies showed that the most important bioactive constituents in $O$. sinensis and its analogs were soluble nucleosides. Since cordycepin (3'-deoxyadenosine) with antitumor activity was isolated from cultured C. militaris in 1950 [25], nucleosides in Cordyceps have become a focus of research. To date, more than ten nucleosides were detected or isolated from this group, such as adenine, adenosine, 2'-deoxyadenosine, 3'-deoxyadenosine, uracil, uridine, 2'-deoxyuridine, guanine, cytosine, guanosine, hypoxanthine, inosine, thymine and thymidine [4,26,27]. Adenosine plays a key role in the pharmacological effects, as it depressed the excitability of CNS neurons and inhibited the release of various presynaptic neurotransmitters [28,29], and adenosine has been used as a marker for quality control of $O$. sinensis in the Chinese Pharmacopoeia [30]. Inosine, the major biochemical metabolite resulting from oxidative deamination of adenosine, stimulated axon growth in vitro and the adult central nervous system [31]. Cordycepin, one of the main compounds found in C. militaris, had also 
shown multiple pharmacological activities [32-34]. However, whether or not natural and cultured $O$. sinensis contain cordycepin is still controversial [27,35-37]. In addition, nucleosides were reported to play a role in growth and differentiation of the gastrointestinal tract, as well as to play a role in the maintenance of the immune response [38,39]. So far, several methods, including HPLC [27,35-37,40,41], LC-MS [26,36,42,43], CE-MS [44], CE [45], CEC [46], ultra-performance liquid chromatography (UPLC) [47], and CZE [48], had been established to determine nucleosides in O. sinensis and related species.

Cordyceps cicadae is recorded as one of the most valued Traditional Chinese Medicines [17]. It consists of the dried fungus Isaria cicadae growing on the nymphs of cicadas. The fruiting body (coremium) and the nymph (sclerotium) of $C$. cicadae have been applied together in Traditional Chinese Medicine and food. Up to now, the nucleosides of coremium and sclerotium have not been determined in populations of $C$. cicadae. In this study, a simple and convenient HPLC method was used to analyze the nucleosides in coremium and sclerotium of $C$. cicadae populations, comparing with those of $O$. sinensis and $C$. militaris. This method included a system of 10 nucleosides, i.e., uracil, uridine, 2'-deoxyuridine, inosine, guanosine, adenine, thymidine, adenosine, 2'-deoxyadenosine and cordycepin (3'-deoxyadenosine). The nucleoside distribution patterns in populations of $C$. cicadae were revealed, and these compounds could become as useful markers for the authentication and quality control of C. cicadae.

\section{Results and Discussions}

Statistical analysis system (SAS) 8.1 was used to analyze the contents of nucleosides in C. cicadae, $O$. sinensis and $C$. militaris. Descriptive statistical analysis, nested analysis and $\mathrm{Q}$ cluster analysis (average cluster) of the data are presented in this paper.

\subsection{Descriptive Statistical Analysis}

The mean content and coefficient of variation $(\mathrm{CV})$ of 10 nucleosides in coremium and sclerotium of $C$. cicadae, O. sinensis and C. militaris are shown in Tables 2 and 3. In coremium, the content of uracil was $279.84-444.47 \mu \mathrm{g} / \mathrm{g}$, the $C V$ was $22.23 \%-40.87 \%$, and the average content was $344.60 \mu \mathrm{g} / \mathrm{g}$. The content of uridine was $363.30-1,928.73 \mu \mathrm{g} / \mathrm{g}$, the $C V$ was $12.93 \%-81.78 \%$, the average content of uridine was $1,468.78 \mu \mathrm{g} / \mathrm{g}$. The content of $2^{\prime}$-deoxyuridine was $49.89-350.41 \mu \mathrm{g} / \mathrm{g}$, the $C V$ was $33.31 \%-95.22 \%$, the average content was $171.24 \mu \mathrm{g} / \mathrm{g}$. The content of inosine was $79.65-1,166.62 \mu \mathrm{g} / \mathrm{g}$, the $C V$ was $10.33 \%-82.36 \%$, the average content was $456.03 \mu \mathrm{g} / \mathrm{g}$. The content of guanosine was $351.44-1,483.06 \mu \mathrm{g} / \mathrm{g}$, the $C V$ was $8.36 \%-58.24 \%$, the average content was $1,016.53 \mu \mathrm{g} / \mathrm{g}$. The content of adenine was $33.63-166.17 \mu \mathrm{g} / \mathrm{g}$, the $C V$ was $32.36 \%-66.30 \%$, the average content was $70.17 \mu \mathrm{g} / \mathrm{g}$. The content of thymidine was $17.73-83.81 \mu \mathrm{g} / \mathrm{g}$, the $C V$ was $29.20 \%-91.67 \%$, the average content was $38.96 \mu \mathrm{g} / \mathrm{g}$. The content of adenosine was $201.54-1,153.78 \mu \mathrm{g} / \mathrm{g}$, the $C V$ was $10.33 \%-56.48 \%$, the average content was $797.92 \mu \mathrm{g} / \mathrm{g}$. The content of 2'-deoxyadenosine was $13.01-54.62 \mu \mathrm{g} / \mathrm{g}$, the $C V$ was $25.13 \%-112.36 \%$, the average content was $28.13 \mu \mathrm{g} / \mathrm{g}$. 
Table 2. The content of 10 nucleosides in 10 populations of $C$. cicadae, four populations of $O$. sinensis and one population of $C$. militairis.

\begin{tabular}{|c|c|c|c|c|c|c|c|c|c|c|c|c|}
\hline \multirow{2}{*}{ Pop } & \multirow{2}{*}{ Position } & \multicolumn{10}{|c|}{ Contents (mean $(\mu \mathrm{g} / \mathrm{g}) / \mathrm{CV}(\%))$} & \multirow{2}{*}{ Total amount $(\mu \mathrm{g} / \mathrm{g})$} \\
\hline & & Uracil & Uridine & 2'-Deoxyuridine & Inosine & Guanosine & Adenine & Thymidine & Adenosine & 2'-deoxyadenosine & Cordycepin & \\
\hline \multirow{2}{*}{ CCKSG } & coremium & $444.47 / 33.73$ & $1577.49 / 19.00$ & $350.41 / 95.22$ & $1166.62 / 10.33$ & $733.03 / 42.09$ & $166.17 / 32.36$ & $83.81 / 29.20$ & $837.00 / 39.93$ & $54.62 / 25.13$ & $-{ }^{\mathrm{b}}$ & 5413.62 \\
\hline & sclerotium & $315.63 / 62.24$ & $774.72 / 41.32$ & $57.24 / 49.62$ & $198.44 / 77.06$ & $314.61 / 48.99$ & $99.15 / 34.95$ & $75.02 / 39.66$ & $510.99 / 74.40$ & $52.94 / 26.47$ & - & 2398.75 \\
\hline \multirow{2}{*}{ CCLHH } & coremium & $334.54 / 27.49$ & $1587.46 / 12.93$ & $119.21 / 40.45$ & $291.28 / 54.31$ & $1330.38 / 12.14$ & $72.39 / 32.70$ & $43.05 / 34.95$ & $857.36 / 21.13$ & $32.81 / 28.25$ & - & 4668.47 \\
\hline & sclerotium & $149.32 / 26.57$ & $1353.86 / 36.23$ & $62.34 / 81.92$ & $98.06 / 44.89$ & $720.48 / 34.92$ & $77.91 / 71.95$ & $138.32 / 126.94$ & $742.77 / 36.52$ & $120.84 / 155.87$ & - & 3463.90 \\
\hline \multirow{2}{*}{ CCLHQ } & coremium & $371.92 / 38.41$ & $1560.08 / 29.22$ & 299.71/70.19 & $490.96 / 65.31$ & $1003.10 / 37.98$ & $67.06 / 44.30$ & $33.06 / 53.97$ & $723.85 / 29.64$ & $15.61 / 66.45$ & - & 4565.36 \\
\hline & sclerotium & $266.09 / 29.50$ & $1221.69 / 17.30$ & $85.03 / 33.91$ & $152.94 / 33.56$ & $648.18 / 18.81$ & $62.09 / 43.60$ & $43.49 / 84.05$ & $575.99 / 16.42$ & $22.74 / 47.99$ & - & 3078.23 \\
\hline \multirow{2}{*}{ CCLHX } & coremium & $351.85 / 22.23$ & $1365.09 / 14.30$ & $100.01 / 41.75$ & $267.39 / 20.71$ & $867.27 / 8.36$ & $58.80 / 32.49$ & $28.27 / 75.87$ & $811.56 / 10.33$ & $25.17 / 42.68$ & - & 3875.42 \\
\hline & sclerotium & $243.02 / 25.84$ & $1224.83 / 17.04$ & $48.13 / 62.70$ & $95.47 / 16.39$ & $626.64 / 10.77$ & $55.06 / 39.08$ & $68.09 / 108.80$ & $629.61 / 11.29$ & $34.13 / 98.29$ & - & 3024.97 \\
\hline \multirow{2}{*}{ CCLSH } & coremium & $279.84 / 40.87$ & $1676.29 / 36.07$ & $88.49 / 61.11$ & $456.96 / 65.69$ & $1091.44 / 40.48$ & $66.65 / 57.11$ & $21.56 / 55.73$ & $727.60 / 39.93$ & $14.31 / 53.31$ & - & 4423.14 \\
\hline & sclerotium & $237.62 / 20.18$ & $1387.93 / 20.31$ & $53.99 / 48.53$ & $181.70 / 43.04$ & $614.19 / 12.73$ & $76.20 / 29.13$ & $45.70 / 44.16$ & $585.88 / 26.66$ & $25.17 / 41.58$ & - & 3208.38 \\
\hline \multirow{2}{*}{ CCLTD } & coremium & $305.86 / 23.19$ & $1278.23 / 21.53$ & $94.33 / 33.31$ & $409.24 / 58.69$ & 751.05/17.91 & $43.21 / 41.17$ & $27.58 / 64.09$ & $749.17 / 21.50$ & $35.72 / 46.07$ & - & 3694.39 \\
\hline & sclerotium & $204.83 / 21.19$ & $1117.27 / 22.69$ & $40.83 / 39.27$ & $159.18 / 52.50$ & $661.92 / 11.23$ & $43.13 / 54.55$ & $52.58 / 73.60$ & $678.05 / 18.48$ & $44.48 / 48.67$ & - & 3002.28 \\
\hline \multirow{2}{*}{ CCLTL } & coremium & $289.52 / 37.70$ & $1460.48 / 21.09$ & $169.42 / 62.02$ & $418.79 / 82.36$ & $1207.12 / 39.58$ & $51.70 / 39.76$ & $17.73 / 91.67$ & $923.07 / 21.93$ & $13.01 / 53.70$ & - & 4550.85 \\
\hline & sclerotium & $191.12 / 28.90$ & $1159.95 / 28.57$ & $63.93 / 53.47$ & $96.83 / 33.11$ & $608.91 / 16.31$ & $61.13 / 100.84$ & $59.21 / 73.47$ & $564.37 / 15.25$ & $32.67 / 57.02$ & - & 2838.13 \\
\hline \multirow{2}{*}{ CCLZD } & coremium & $404.82 / 23.43$ & $1928.73 / 19.57$ & $263.10 / 84.10$ & $587.61 / 66.12$ & $1347.46 / 36.69$ & $75.06 / 66.30$ & $45.65 / 60.17$ & $994.31 / 27.95$ & $31.48 / 47.22$ & - & 5678.21 \\
\hline & sclerotium & $261.29 / 23.35$ & 1587.68/26.09 & $80.34 / 50.33$ & $179.66 / 42.85$ & $802.61 / 14.57$ & $60.07 / 43.57$ & $91.87 / 74.81$ & $715.34 / 13.00$ & $55.68 / 43.89$ & - & 3834.54 \\
\hline \multirow{2}{*}{ CCWYJ } & coremium & $334.14 / 38.45$ & $1890.68 / 35.48$ & $177.81 / 85.93$ & $391.80 / 61.14$ & $1483.06 / 36.78$ & $67.02 / 43.24$ & $49.37 / 40.09$ & $1153.78 / 37.73$ & $42.67 / 39.45$ & - & 5590.34 \\
\hline & sclerotium & $207.19 / 25.52$ & $1516.08 / 28.42$ & $40.94 / 81.69$ & $105.91 / 43.88$ & $859.40 / 25.08$ & $52.03 / 40.70$ & $101.56 / 62.21$ & $961.26 / 23.62$ & $97.28 / 67.09$ & - & 3941.64 \\
\hline \multirow{2}{*}{ CCYTS } & coremium & $329.00 / 39.83$ & $363.30 / 81.78$ & $49.89 / 78.96$ & $79.65 / 72.59$ & $351.44 / 58.24$ & $33.63 / 44.80$ & $39.51 / 51.17$ & $201.54 / 56.48$ & $15.94 / 112.36$ & - & 1463.89 \\
\hline & sclerotium & $187.25 / 32.74$ & $520.61 / 19.82$ & $23.97 / 44.95$ & $74.61 / 42.69$ & $260.00 / 30.74$ & $33.93 / 32.31$ & $29.55 / 77.86$ & $230.20 / 35.14$ & $9.68 / 89.86$ & - & 1369.80 \\
\hline \multirow{2}{*}{ OSDQI } & stroma & $353.39 / 45.17$ & $2276.38 / 23.94$ & $6.67 / 138.48$ & $155.27 / 22.06$ & $1599.02 / 9.48$ & $178.67 / 65.91$ & $63.85 / 15.68$ & $1685.50 / 8.50$ & $47.01 / 18.41$ & - & 6365.76 \\
\hline & sclerotium & $213.93 / 60.96$ & $1432.24 / 22.90$ & $16.76 / 136.97$ & $430.95 / 9.82$ & $864.00 / 44.40$ & $91.23 / 25.40$ & $124.32 / 19.25$ & $675.15 / 70.58$ & $49.26 / 25.25$ & - & 3897.84 \\
\hline \multirow{2}{*}{ OSLTA } & stroma & $55.31 / 24.31$ & $1572.67 / 13.23$ & - & $141.80 / 50.78$ & $1176.66 / 10.46$ & $79.75 / 24.11$ & $24.03 / 44.75$ & $1388.17 / 11.95$ & $46.75 / 22.65$ & - & 4485.14 \\
\hline & sclerotium & $48.18 / 43.55$ & 1468.93/19.52 & - & $615.71 / 20.66$ & $978.12 / 4.53$ & $62.08 / 29.11$ & $69.50 / 24.76$ & $451.90 / 47.68$ & $52.18 / 33.81$ & - & 3746.60 \\
\hline \multirow{2}{*}{ OSMNI } & stroma & $170.45 / 47.78$ & $1387.75 / 7.94$ & - & $96.01 / 40.87$ & $1481.03 / 8.16$ & $108.00 / 17.00$ & $52.05 / 29.99$ & $1619.77 / 12.70$ & $44.54 / 32.96$ & - & 4959.60 \\
\hline & sclerotium & $116.40 / 15.01$ & $1255.39 \pm / 5.47$ & - & $399.60 / 12.43$ & $1070.80 / 3.27$ & $99.19 / 24.49$ & $147.95 / 12.60$ & $752.39 / 8.92$ & $58.51 / 17.55$ & - & 3900.23 \\
\hline
\end{tabular}


Table 2. Cont.

\begin{tabular}{|c|c|c|c|c|c|c|c|c|c|c|c|c|}
\hline \multirow{2}{*}{ Pop } & \multirow{2}{*}{ Position } & \multicolumn{10}{|c|}{ Contents $($ mean $(\mu \mathrm{g} / \mathrm{g}) / \mathrm{CV}(\%))$} & \multirow{2}{*}{ Total amount $(\mu \mathrm{g} / \mathrm{g})$} \\
\hline & & Uracil & Uridine & 2'-Deoxyuridine & Inosine & Guanosine & Adenine & Thymidine & Adenosine & 2'-deoxyadenosine & Cordycepin & \\
\hline \multirow{2}{*}{ OSNBE } & stroma & $149.76 / 11.15$ & $2765.61 / 2.92$ & $7.70 / 19.07$ & $580.85 / 17.06$ & $2130.34 / 3.59$ & $113.59 / 21.67$ & $58.34 / 26.80$ & $2544.76 / 4.02$ & $46.77 / 24.73$ & - & 8397.72 \\
\hline & sclerotium & $127.95 / 15.59$ & $1599.98 / 6.53$ & $9.92 / 27.35$ & $2073.63 / 18.43$ & $1131.88 / 4.76$ & $154.86 / 11.56$ & $177.09 / 9.04$ & $1081.60 / 8.63$ & $106.28 / 17.61$ & - & 6463.19 \\
\hline \multirow{2}{*}{ CMSMB } & stroma & $319.18 / 19.51$ & $1900.92 / 11.02$ & $5.01 / 24.88$ & $85.08 / 20.14$ & $1215.38 / 16.31$ & $313.75 / 20.12$ & $69.41 / 14.69$ & $1613.28 / 13.51$ & $58.18 / 19.15$ & $659.29 / 19.11$ & 6239.49 \\
\hline & sclerotium & $332.93 / 20.04$ & $1743.60 / 13.87$ & $12.77 / 18.48$ & $189.93 / 13.14$ & $1075.99 / 16.58$ & $264.18 / 20.17$ & $68.87 / 16.74$ & $1655.93 / 12.37$ & $76.98 / 15.84$ & $4173.57 / 13.81$ & 9594.75 \\
\hline
\end{tabular}

Table 3. The average content $(\mu \mathrm{g} / \mathrm{g})$ and $C V(\%)$ of 10 nucleosides in C. cicadae, O. sinensis and C. militairis.

\begin{tabular}{|c|c|c|c|c|c|c|c|c|c|c|c|c|}
\hline \multirow{2}{*}{ Species } & \multirow{2}{*}{ Position } & \multicolumn{10}{|c|}{ Mean content $(\mu \mathrm{g} / \mathrm{g}) / \mathrm{CV}(\%)$} & \multirow{2}{*}{ Total amount $(\mu \mathrm{g} / \mathrm{g})$} \\
\hline & & Uracil & Uridine & 2'-Deoxyuridine & Inosine & Guanosine & Adenine & Thymidine & Adenosine & 2'-deoxyadenosine & Cordycepin & \\
\hline C. cicadae & coremium & $344.60 / 34.66$ & $1468.78 / 38.58$ & $171.24 / 104.35$ & $456.03 / 79.55$ & $1016.53 / 46.92$ & $70.17 / 65.99$ & $38.96 / 66.95$ & $797.92 / 42.21$ & $28.13 / 64.84$ & $-{ }^{b}$ & 4392.37 \\
\hline$\left(\mathrm{n}^{\mathrm{c}}=10\right)$ & sclerotium & $226.34 / 40.18$ & $1186.46 / 37.05$ & $55.68 / 63.15$ & $134.28 / 60.32$ & $611.69 / 36.79$ & $62.07 / 59.85$ & $70.54 / 106.70$ & $619.45 / 40.64$ & $49.56 / 142.54$ & - & 3016.06 \\
\hline O. sinensis & stroma & $182.23 / 75.88$ & $2000.60 / 31.48$ & $3.59 / 157.80$ & $243.48 / 86.32$ & $1596.76 / 23.20$ & $120.00 / 56.37$ & $49.57 / 40.12$ & $1809.55 / 26.14$ & $46.27 / 23.07$ & - & 6052.06 \\
\hline$(n=4)$ & sclerotium & $126.61 / 68.28$ & $1439.14 / 16.90$ & $6.67 / 192.88$ & $879.97 / 83.68$ & $1011.20 / 20.51$ & $101.84 / 38.79$ & $129.71 / 34.04$ & $740.26 / 45.62$ & $66.56 / 41.41$ & - & 4501.96 \\
\hline C. militaris & stroma & $319.18 / 19.51$ & 1900.92/11.02 & $5.01 / 24.88$ & $85.08 / 20.14$ & $1215.38 / 16.31$ & $313.75 / 20.12$ & $69.41 / 14.69$ & $1613.28 / 13.51$ & $58.18 / 19.15$ & $659.29 / 19.11$ & 6239.49 \\
\hline$(\mathrm{n}=1)$ & sclerotium & $332.93 / 20.04$ & $1743.60 / 13.87$ & $12.77 / 18.48$ & $189.93 / 13.14$ & $1075.99 / 16.58$ & $264.18 / 20.17$ & $68.87 / 16.74$ & $1655.93 / 12.37$ & $76.98 / 15.84$ & $4173.57 / 13.81$ & 9594.75 \\
\hline
\end{tabular}

${ }^{\mathrm{b}}$ Not detected; ${ }^{\mathrm{c}}$ Number of population. 
In sclerotium, the content of uracil was $149.32-315.63 \mu \mathrm{g} / \mathrm{g}$, the $C V$ was $20.18 \%-62.24 \%$, the average content was $226.34 \mu \mathrm{g} / \mathrm{g}$. The content of uridine was $520.61-1587.68 \mu \mathrm{g} / \mathrm{g}$, the $C V$ was $17.04 \%-41.32 \%$, the average content of uridine was $1,186.46 \mu \mathrm{g} / \mathrm{g}$. The content of 2 -deoxyuridine was $23.97-85.03 \mu \mathrm{g} / \mathrm{g}$, the $C V$ was $33.91 \%-81.92 \%$, the average content was $55.68 \mu \mathrm{g} / \mathrm{g}$. The content of inosine was $74.61-198.44 \mu \mathrm{g} / \mathrm{g}$, the $C V$ was $16.39 \%-77.06 \%$, the average content was $134.28 \mu \mathrm{g} / \mathrm{g}$. The content of guanosine was $260.00-859.40 \mu \mathrm{g} / \mathrm{g}$, the $C V$ was $10.77 \%-48.99 \%$, the average content was $611.69 \mu \mathrm{g} / \mathrm{g}$. The content of adenine was $33.93-99.15 \mu \mathrm{g} / \mathrm{g}$, the $C V$ was $29.13 \%-100.84 \%$, the average content was $62.07 \mu \mathrm{g} / \mathrm{g}$. The content of thymidine was $29.55-138.32 \mu \mathrm{g} / \mathrm{g}$, the $C V$ was $39.66 \%-126.94 \%$, the average content was $70.54 \mu \mathrm{g} / \mathrm{g}$. The content of adenosine was $230.20-961.26 \mu \mathrm{g} / \mathrm{g}$, the $C V$ was $11.29 \%-74.40 \%$, the average content was $619.45 \mu \mathrm{g} / \mathrm{g}$. The content of 2 -deoxyadenosine was $9.68-120.84 \mu \mathrm{g} / \mathrm{g}$, the $C V$ was $26.47 \%-155.87 \%$, the average content was $49.56 \mu \mathrm{g} / \mathrm{g}$.

Analysis of the nucleosides revealed obvious differences between coremium and sclerotium in populations of $C$. cicadae. The contents of uracil, uridine, 2'-deoxyuridine, inosine, guanosine, adenine and adenosine in coremium were higher than those in sclerotium. The coefficient of variation in coremium was $34.66 \%-104.35 \%$, and the $C V$ in sclerotium was $36.79 \%-142.54 \%$, with a great variation of nucleosides content in populations of $C$. cicadae. The wide variation of nucleosides in C. cicadae populations may mainly be derived from the genetic differences of the C. cicadae population, being affected by different location, geography, climate, maturation of the $C$. cicadae. Furthermore, Li et al. reported that after storage of $O$. sinensis at $75 \%$ relative humidity and $40{ }^{\circ} \mathrm{C}$ for 10 days, the contents of uridine, guanosine and adenosine in natural $O$. sinensis were markedly increased about one to four fold [49], implying that the storage conditions might be another factor affecting the variation of nucleosides in C. cicadae.

Nucleosides were believed to be the active components in Cordyceps-like fungi [50], indeed, Cordyceps-like fungi contained a higher concentration of nucleosides [51], and some unique nucleosides, such as cordycepin, 2'-deoxyuridine and 2'-deoxyadenosine were detected in Cordyceps-like fungus [26,27,45,47,51-53], which could be used as markers for distinguishing Cordyceps-like fungi from their counterfeits.

Cordycepin in natural $O$. sinensis was found in very low amounts [36,46,54], about several tens of micrograms per gram [55]. However, in this study, cordycepin was not detected in C. cicadae and O. sinensis, and cordycepin in C. militaris was high, up to $659.29 \mu \mathrm{g} / \mathrm{g}$ in stroma and $4173.57 \mu \mathrm{g} / \mathrm{g}$ in sclerotium, in accordance with the reports of Guo et al., and Yang and Li [36,37]. 2'-Deoxyadenosine was detected in C. cicadae, i.e., $28.13 \mu \mathrm{g} / \mathrm{g}$ in coremium and $49.56 \mu \mathrm{g} / \mathrm{g}$ in sclerotium. Cordycepin and 2'-deoxyadenosine are isomers of each other, and there are a lot of reports about the pharmacological activities of cordycepin [32-34], while the pharmacological activities of 2'-deoxyadenosine in Cordyceps-like fungi are worth studying further.

$\mathrm{Li}$ et al. reported that the levels of adenosine, guanosine and uridine were very similar in stroma and sclerotium of $O$. sinensis [54]. The average content of nucleosides of 10 populations of $C$. cicadae, four populations of $O$. sinensis and one population of $C$. militaris are shown in Table 3 . Several nucleosides such as uracil, uridine, guanosine, adenine and adenosine in coremium were higher than those in sclerotium of $C$. cicadae. On the contrary, the content of thymidine and 2'-deoxyadenosine in coremium were lower than those in sclerotium of C. cicadae. The average 
content of inosine in coremium $(456.03 \mu \mathrm{g} / \mathrm{g})$ was 3-fold higher than that in sclerotium $(134.28 \mu \mathrm{g} / \mathrm{g})$ of C. cicadae.

Hsu et al. reported that the content of adenosine in stroma was approximately 6-fold higher than that in sclerotium of $O$. sinensis [56]. However, the content of adenosine in coremium was approximately 1.5 times that in sclerotium of $C$. cicadae. The total content of the 10 nucleosides in coremium was approximately 1.5 times that in sclerotium in $C$. cicadae. On the contrary, the content of the 10 analytes in stroma was approximately 1.5 times that in sclerotium of $C$. militaris. The distribution of the 10 nucleosides in $C$. cicadae was similar to that in $O$. sinensis, being different from the distribution pattern of the 10 analytes in C. militaris.

\subsection{Nested Analysis}

Nested analysis was used to analyze the uracil, uridine, 2'-deoxyuridine, inosine, guanosine, adenine, thymidine, adenosine and 2'-deoxyadenosine in C. cicadae, investigating the percent of total variance of the nine analytes between populations and individuals.

The result of nested variation analysis by the nine nucleosides' distributions is shown in Table 4 . It was indicated that about $42.29 \%$ of the variation in coremium was attributed to the differentiation among populations, and the remaining $57.71 \%$ was resided among individuals within populations. It was also showed that about $28.94 \%$ of the variation in sclerotium was expressed between populations, while most of the variation $71.06 \%$ was resided among individuals within populations.

Table 4. Nested analysis of nine nucleosides in coremium and sclerotium of C. cicadae.

\begin{tabular}{|c|c|c|c|c|c|c|}
\hline Position & Analyte & $\begin{array}{l}\text { Percent of total } \\
\text { variance }(\%)\end{array}$ & $\begin{array}{c}\text { Percent of population } \\
\text { variance }(\%)\end{array}$ & $\begin{array}{c}\text { Percent of individual } \\
\text { variance }(\%)\end{array}$ & F Value & $\operatorname{Pr}>F$ \\
\hline \multirow{10}{*}{ coremium } & uracil & 100.00 & 9.13 & 90.87 & 2.00 & 0.0477 \\
\hline & uridine & 100.00 & 52.45 & 47.55 & 12.03 & $<0.0001$ \\
\hline & 2'-deoxyuridine & 100.00 & 23.83 & 76.17 & 4.13 & 0.0002 \\
\hline & inosine & 100.00 & 54.48 & 45.52 & 12.97 & $<0.0001$ \\
\hline & guanosine & 100.00 & 45.25 & 54.75 & 9.27 & $<0.0001$ \\
\hline & adenine & 100.00 & 53.57 & 46.43 & 12.54 & $<0.0001$ \\
\hline & thymidine & 100.00 & 44.82 & 55.18 & 9.12 & $<0.0001$ \\
\hline & adenosine & 100.00 & 46.69 & 53.31 & 9.76 & $<0.0001$ \\
\hline & 2'-deoxyadenosine & 100.00 & 50.39 & 49.61 & 11.16 & $<0.0001$ \\
\hline & Mean & 100.00 & 42.29 & 57.71 & $\longrightarrow$ & - \\
\hline \multirow{10}{*}{ sclerotium } & uracil & 100.00 & 19.25 & 80.75 & 3.38 & 0.0013 \\
\hline & uridine & 100.00 & 47.52 & 52.48 & 10.06 & $<0.0001$ \\
\hline & 2'-deoxyuridine & 100.00 & 19.19 & 80.81 & 3.37 & 0.0013 \\
\hline & inosine & 100.00 & 22.04 & 77.96 & 3.83 & 0.0004 \\
\hline & guanosine & 100.00 & 63.70 & 36.30 & 18.55 & $<0.0001$ \\
\hline & adenine & 100.00 & 16.48 & 83.52 & 2.97 & 0.0038 \\
\hline & thymidine & 100.00 & 9.45 & 90.55 & 2.04 & 0.0432 \\
\hline & adenosine & 100.00 & 47.44 & 52.56 & 10.03 & $<0.0001$ \\
\hline & 2'-deoxyadenosine & 100.00 & 15.38 & 84.62 & 2.82 & 0.0058 \\
\hline & Mean & 100.00 & 28.94 & 71.06 & - & - \\
\hline
\end{tabular}




\subsection{Q Cluster Analysis}

O. sinensis, one of the most precious Traditional Chinese Medicines grows in a very restricted habitat, and is usually found in the soil of prairies or fir forests at an altitude from 3,500 to 5,000 m, mainly in provinces like Sichuan, Qinghai, Yunnan, Tibet and Gansu in China. In Nepal, Bhutan and India, O. sinensis is collected as well. In China, this fungus is usually called "Dong Chong Xia Cao". $O$. sinensis has been used for the treatment of hyperglycemia, respiratory and liver diseases, renal dysfunction, renal failure and has antioxidant properties [57,58]. It was initially recorded in Ben-CaoBei-Yao by Wang Ang in 1694. Because of its scarcity in nature and high price, some studies have been carried out in order to find substitutes for $O$. sinensis $[49,59,60]$. C. militaris have been used as the main substitute for $O$. sinensis [50,61], and Traditional Chinese Medicine considers C. cicadae to be a drug similar to $O$. sinensis, as these two species have similar active components and medicinal value [17], however, little scientific information about the proximate composition and bioactive ingredients of C. cicadae and $O$. sinensis is available. Q cluster analysis (average linkage) was used to analyze the 10 nucleosides in C. cicadae, O. sinensis and C. militaris (Figures 3 and 4).

Figure 3. Q-Cluster of 10 nucleosides assayed in coremium (stroma) of 10 populations of $C$. cicadae, four populations of $O$. sinensis, and one population of $C$. militaris, using the average linkage method.

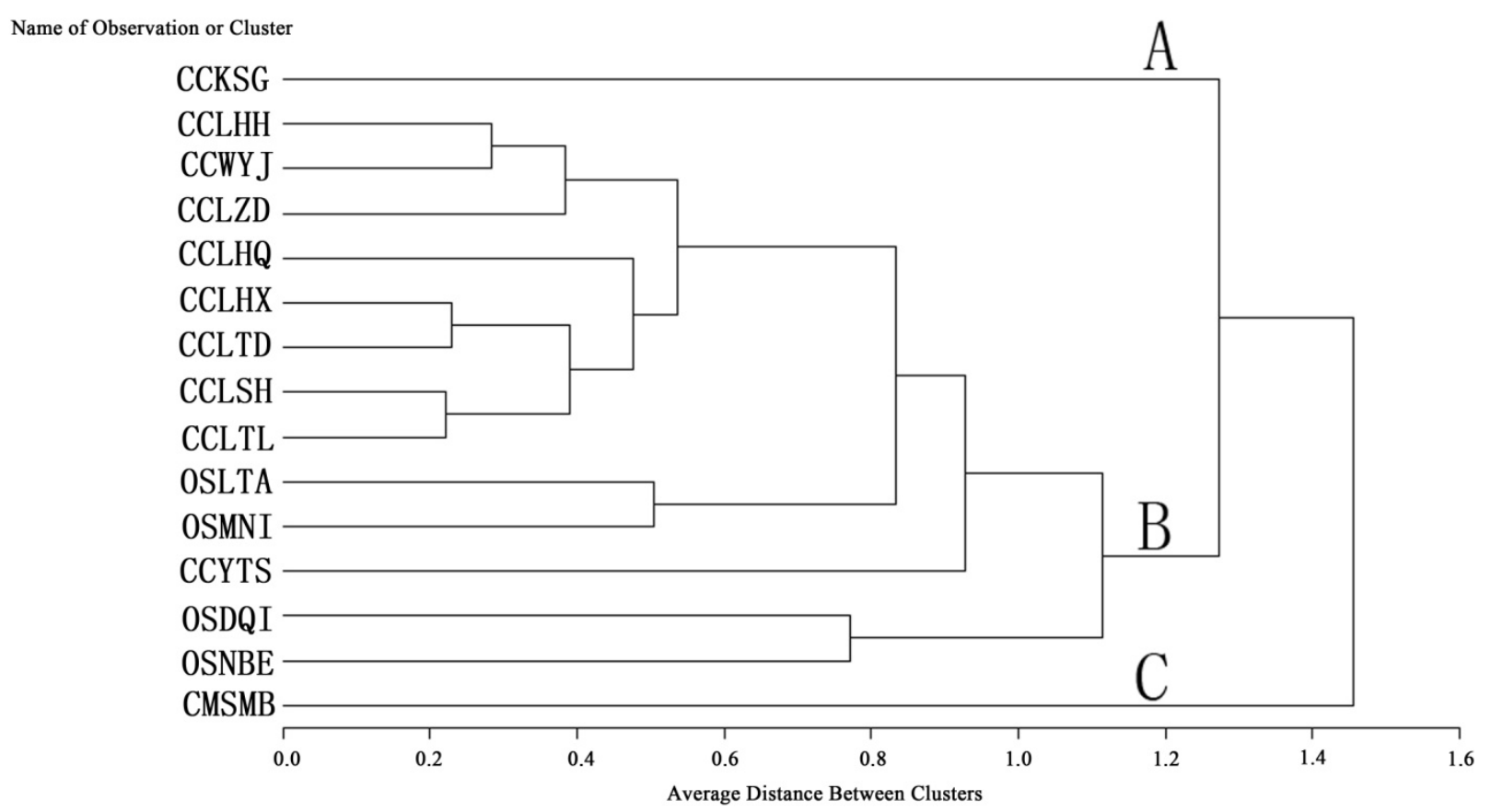

Figure 3 shows the 15 populations of $C$. cicadae, $O$. sinensis and $C$. militaris separated into three branches; clade A includes populatation CCKSG, clade $\mathrm{C}$ includes population CMSMB, and clade B includes all other populations. The populations of $C$. cicadae collected at Lanping and Weixi county clustered as one subclade, which showed the geological differences. In Figure 4, 15 populations of C. cicadae, $O$. sinensis and C. militaris also separate into three branches; clade D includes 10 populations of $C$. cicadae and three populations of $O$. sinensis, clade E includes population OSNBE, and clade $\mathrm{F}$ includes population CMSMB. 
Figure 4. Q-Cluster of 10 nucleosides assayed in sclerotium of 10 populations of C. cicadae, four populations of $O$. sinensis, and one population of $C$. militaris by the average linkage method.

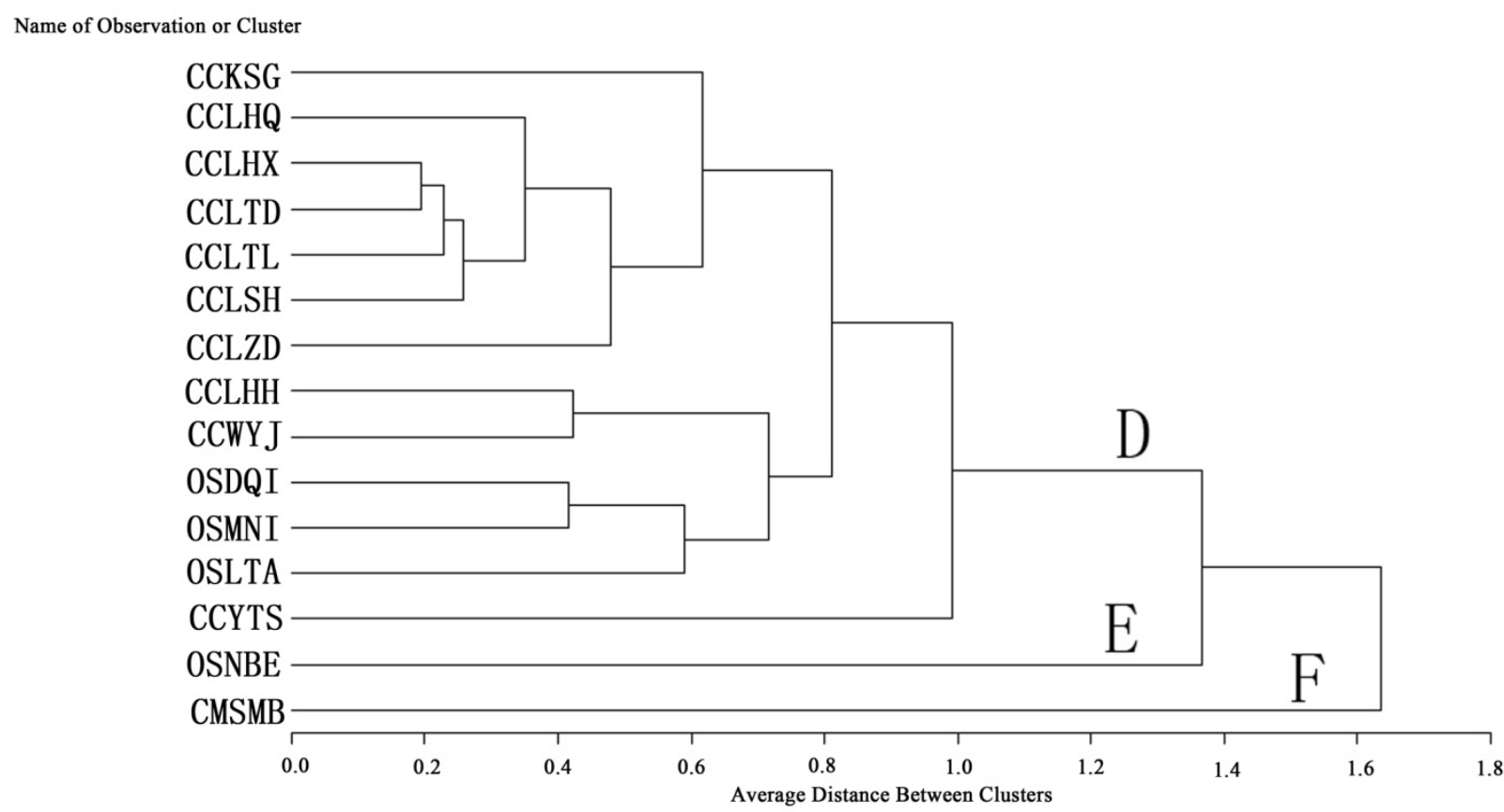

The four populations of $O$. sinensis could not be separated into a single clade, indicating that the nucleosides in $O$. sinensis had no obvious differences from those of $C$. cicadae. The average clusters based on the average content of uracil, uridine, 2'-deoxyuridine, inosine, guanosine, thymidine, adenine, adenosine, 2'-deoxyadenosine and cordycepin had been constructed, showing that $C$. cicadae should be a better substitute for $O$. sinensis than $C$. militaris.

\section{Experimental}

\subsection{Sample Preparation}

The details of the sources of $C$. cicadae, O. sinensis and C. militaris, are shown in Table 5 . The samples, divided into the fruiting body (coremium or stroma) and the nymph or caterpillar (sclerotium), were dried at $50 \mathrm{C}$ and ground into powder. These were separately weighed into a $5 \mathrm{~mL}$ volumetric flask, $20 \%$ methanol was added to the flask to about $90 \%$ of its volume, and after sonication for $90 \mathrm{~min}$, the mixture was diluted to the mark with $20 \%$ methanol. After centrifugation at $25 \mathrm{C}$ for $10 \mathrm{~min}$ at $4,000 \mathrm{rpm} / \mathrm{min}$, sample solutions were passed through a $0.45 \mu \mathrm{m}$ membrane filter. Duplicate analytical samples were prepared for each sample. The HPLC chromatograms of C. cicadae and mixed standards are shown in Figure 5. 
Table 5. Localities of the 10 populations of C. cicadae, four populations of $O$. sinensis and one population of C. militaris.

\begin{tabular}{|c|c|c|c|c|}
\hline Species & NO. of populations & Samlpe size & Locus of extraction & Locality \\
\hline \multirow{20}{*}{ C. cicadae } & \multirow{2}{*}{ CCKSG } & 10 & Coremium & \multirow{2}{*}{ Gelezicun, Shuanglong township, Kunming City, Yunnan } \\
\hline & & 10 & Sclerotium & \\
\hline & \multirow{2}{*}{ CCLHH } & 10 & Coremium & \multirow{2}{*}{ Hedongqingcun, Hexi township, Lanping county, Yunan } \\
\hline & & 10 & Sclerotium & \\
\hline & \multirow{2}{*}{ CCLHQ } & 10 & Coremium & \multirow{2}{*}{ Qidenglongcun, Hexi township, Lanping county, Yunan } \\
\hline & & 10 & Sclerotium & \\
\hline & \multirow{2}{*}{ CCLHX } & 10 & Coremium & \multirow{2}{*}{ Xiaqingtoucun, Hexi township, Lanping county, Yunan } \\
\hline & & 10 & Sclerotium & \\
\hline & \multirow{2}{*}{ CCLSH } & 10 & Coremium & \multirow{2}{*}{ Huilongcun, Shideng township, Lanping county, Yunan } \\
\hline & & 10 & Sclerotium & \\
\hline & \multirow{2}{*}{ CCLTD } & 10 & Coremium & \multirow{2}{*}{ Deqingcun, Tongdian township, Lanping county, Yunan } \\
\hline & & 10 & Sclerotium & \\
\hline & \multirow{2}{*}{ CCLTL } & 10 & Coremium & \multirow{2}{*}{ Lianqiaoshacun, Tongdian township, Lanping county, Yunan } \\
\hline & & 10 & Sclerotium & \\
\hline & \multirow{2}{*}{ CCLZD } & 10 & Coremium & \multirow{2}{*}{ Datujicun, Zhongpai township, Langping county,Yunnan } \\
\hline & & 10 & Sclerotium & \\
\hline & \multirow{2}{*}{ CCWYJ } & 10 & Coremium & \multirow{2}{*}{ Juxiangcun, Yongchun township, Weixi county, Yunan } \\
\hline & & 10 & Sclerotium & \\
\hline & \multirow{2}{*}{ CCYTS } & 10 & Coremium & \multirow{2}{*}{ Sanzhou Mountain, Taihua town, Yixing city,Jiangsu } \\
\hline & & 10 & Sclerotium & \\
\hline \multirow{8}{*}{ O. sinensis } & \multirow{2}{*}{ OSDQI } & 5 & Stroma & \multirow{2}{*}{ Deqin, county, Yunnan } \\
\hline & & 5 & Sclerotium & \\
\hline & \multirow{2}{*}{ OSMNI } & 5 & Stroma & \multirow{2}{*}{ Manicun, Lengda township, Jiacha county, Tibet } \\
\hline & & 5 & Sclerotium & \\
\hline & \multirow{2}{*}{ OSLTA } & 5 & Stroma & \multirow{2}{*}{ Litang county, Sichuan } \\
\hline & & 5 & Sclerotium & \\
\hline & \multirow{2}{*}{ OSNBE } & 5 & Stroma & \multirow{2}{*}{ Nepal } \\
\hline & & 5 & Sclerotium & \\
\hline \multirow{2}{*}{ C. militaris } & CMSMB & 5 & Stroma & Baivi townshin Sonoming county Yunnan \\
\hline & & 5 & Sclerotium & Daly r townsmp, songmming county, r umman \\
\hline
\end{tabular}


Figure 5. HPLC chromatograms of (A) C. cicadae (Pop CCLTL); (B) mixed standards; 1: uracil; 2: uridine; 3: 2'-deoxyuridine; 4: inosine; 5: guanosine; 6: adenine; 7: thymidine; 8: adenosine; 9: 2'-deoxyadenosine; 10: cordycepin (3'-deoxyadenosine).
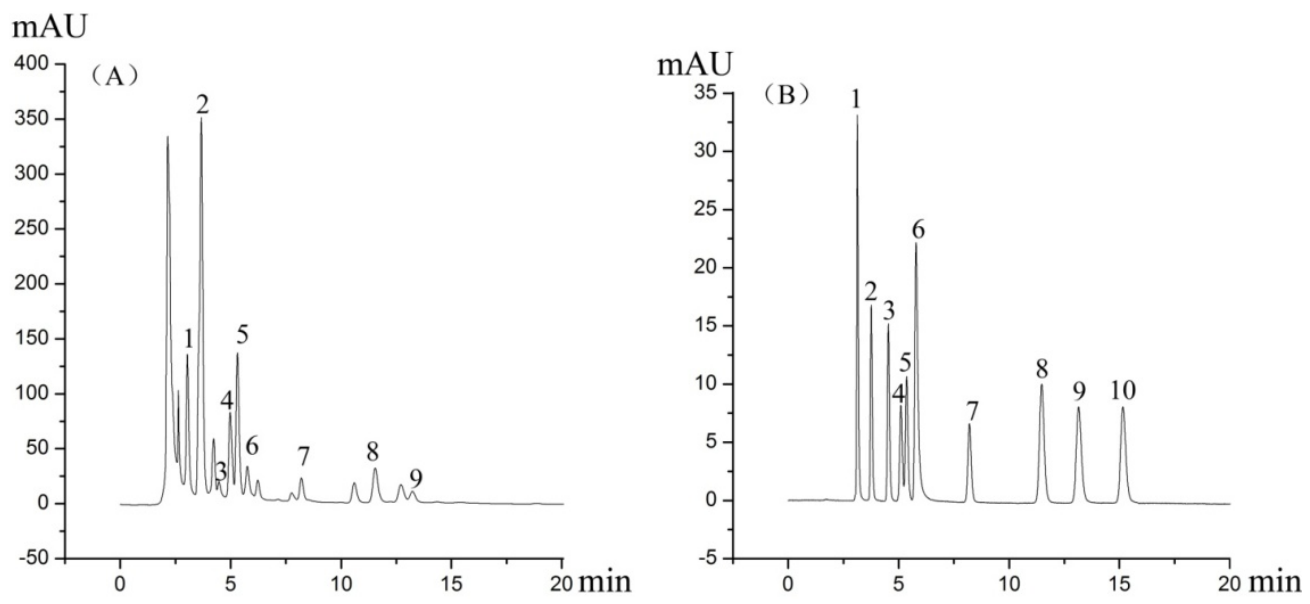

\subsection{Chemicals and Reagents}

HPLC-grade methanol was obtained from Merck KGaA (Darmstadt, Germany). Water was purified using a Millipore Simplicity system (Billerica, MA, USA). Uracil, uridine, 2'-deoxyuridine, inosine, guanosine, thymidine, adenine, adenosine, $2^{\prime}$-deoxyadenosine and cordycepin (purity $\geq 98.0 \%$ ) were purchased from Sigma (St. Louis, MO, USA).

\subsection{Liquid Chromatography Conditions}

HPLC was conducted on a Dionex liquid chromatograph system (DIONEX, Sunnyvale, CA, USA) equipped with a LPG-3400A quaternary pump and a PDA-3000 photodiode array detector. The sample extracts were separated and analyzed using a Waters Symmetry ${ }^{\circledR} \mathrm{C} 18$ column $(250 \mathrm{~mm}, 4.6 \mathrm{~mm}, 5 \mu \mathrm{m})$ at $30{ }^{\circ} \mathrm{C}$. The mobile phase consisted of $10 \%$ solvent A (methanol) and $90 \%$ solvent B (water). The flow rate was $1.0 \mathrm{~mL} \cdot \mathrm{min}^{-1}$. The detecting wavelength was set between 190 and $380 \mathrm{~nm}$, and the chromatographic peaks were measured at a wavelength of $260 \mathrm{~nm}$ for the detection of nucleosides.

\subsection{Method Validation}

\subsubsection{Calibration Curves}

Stock solutions were prepared by dissolving the standards in $20 \%$ methanol to give $1-2 \mathrm{mg} / \mathrm{mL}$ for uracil, uridine, 2'-deoxyuridine, inosine, guanosine, adenine, thymidine, adenosine, 2'-deoxyadenosine and cordycepin respectively. Further dilution with $20 \%$ methanol was performed to prepare the standard solutions for calibration curves. At least six concentrations of the solution were analyzed in triplicate, and then the calibration curves were constructed by plotting the peak areas versus the concentration of each analyte. The results were shown in Table 6. 
Table 6. Linear regression data, LOD, and LOQ of 10 nucleosides at $260 \mathrm{~nm}$.

\begin{tabular}{lllllll}
\hline Analyte & $\begin{array}{l}\boldsymbol{\lambda} \mathbf{m a x} \\
(\mathbf{n m})\end{array}$ & $\begin{array}{l}\text { Linear Regression } \\
\text { Equation }\end{array}$ & $\mathbf{r}^{2}$ & $\begin{array}{l}\text { Test Range } \\
(\boldsymbol{\mu g} / \mathbf{m L})\end{array}$ & $\begin{array}{l}\text { LOD } \\
(\boldsymbol{\mu g} / \mathbf{m L})\end{array}$ & $\begin{array}{l}\text { LOQ } \\
(\boldsymbol{\mu g} / \mathbf{m L})\end{array}$ \\
\hline uracil & 260.1 & $\mathrm{y}=0.0145 \mathrm{x}-0.0076$ & 0.9999 & $8.00-240.00$ & 0.006 & 0.018 \\
uridine & 263.1 & $\mathrm{y}=0.0233 \mathrm{x}+0.008$ & 0.9999 & $8.00-240.00$ & 0.008 & 0.024 \\
2'-deoxyuridine & 263.2 & $\mathrm{y}=0.0234 \mathrm{x}-0.0016$ & 0.9999 & $7.00-140.00$ & 0.008 & 0.024 \\
inosine & 249.8 & $\mathrm{y}=0.0368 \mathrm{x}-0.0479$ & 0.9995 & $7.60-380.00$ & 0.015 & 0.045 \\
guanosine & 254.2 & $\mathrm{y}=0.023 \mathrm{x}+0.029$ & 0.9997 & $8.00-400.00$ & 0.008 & 0.024 \\
adenine & 261.5 & $\mathrm{y}=0.0108 \mathrm{x}$ & 0.9999 & $8.00-400.00$ & 0.004 & 0.012 \\
thymidine & 268.2 & $\mathrm{y}=0.0315 \mathrm{x}+0.0009$ & 0.9999 & $8.60-172.00$ & 0.012 & 0.036 \\
adenosine & 261.0 & $\mathrm{y}=0.0178 \mathrm{x}-0.0009$ & 0.9999 & $8.40-420.00$ & 0.006 & 0.018 \\
2'-deoxyadenosine & 261.1 & $\mathrm{y}=0.0171 \mathrm{x}+0.0025$ & 0.9999 & $8.40-420.00$ & 0.006 & 0.018 \\
cordycepin & 261.2 & $\mathrm{y}=0.0189 \mathrm{x}-0.0005$ & 0.9999 & $8.40-420.00$ & 0.007 & 0.021 \\
\hline
\end{tabular}

\subsubsection{Limits of Detection and Quantification}

The stock solution containing ten reference compounds was diluted to a series of appropriate concentrations with the same solvent, and an aliquot of the diluted solutions were injected into HPLC for analysis. The limits of detection (LOD) and quantification (LOQ) under the present chromatographic conditions were determined at a signal-to-noise ratio $(\mathrm{S} / \mathrm{N})$ of about 3 and 10 , respectively. The LOD and LOQ data for each compound investigated were shown in Table 6 . The identification of investigated compounds was carried out by comparison of their retention times and UV spectra with those obtained injecting standards in the same conditions or by spiking Cordyceps samples with stock standard solutions.

\subsubsection{Reproducibility and Accuracy}

Reproducibility and accuracy were determined for 10 standard samples at a certain concentration, which was described in Table 7. The intra-day coefficients of variation for the 10 analytes were $0.65 \%-2.49 \%$. The inter-day coefficients of variation for the 10 analytes were $1.08 \%-2.12 \%$. The accuracy $(\%)$ of the method was expressed as the mean deviation of all repetitions from the nominal value. The intra-day accuracy for the 10 analytes was $98.98 \%-101.38 \%$. The inter-day accuracy for the 10 analytes was $98.88 \%-101.53 \%$.

Table 7. Reproducibility and accuracy analysis of 10 nucleosides $(\mathrm{n}=5)$.

\begin{tabular}{ccccc}
\hline Analyte & $\begin{array}{c}\text { Nominal } \\
\text { Concentration }(\boldsymbol{\mu g} / \mathbf{m L})\end{array}$ & $\begin{array}{c}\text { Assay Value }(\mathbf{m e a n} \pm \\
\mathbf{S D})(\boldsymbol{\mu g} / \mathbf{m L})\end{array}$ & $\begin{array}{c}\text { Coefficient of } \\
\text { Variation }(\mathbf{\%})\end{array}$ & $\begin{array}{c}\text { Accuracy } \\
(\mathbf{\%})\end{array}$ \\
\hline intra-day ${ }^{\mathrm{d}}$ & & & & \\
uracil & 40.00 & $39.84 \pm 0.50$ & 1.26 & 99.60 \\
uridine & 40.00 & $39.65 \pm 0.88$ & 2.22 & 99.13 \\
2'-deoxyuridine & 70.00 & $70.71 \pm 1.37$ & 1.94 & 101.01 \\
inosine & 38.00 & $37.65 \pm 0.80$ & 2.12 & 99.08 \\
guanosine & 40.00 & $40.55 \pm 1.01$ & 2.49 & 101.38 \\
adenine & 40.00 & $39.59 \pm 0.97$ & 2.45 & 98.98 \\
thymidine & 86.00 & $85.32 \pm 1.15$ & 1.35 & 99.21 \\
\hline
\end{tabular}


Table 7. Cont.

\begin{tabular}{ccccc}
\hline Analyte & $\begin{array}{c}\text { Nominal } \\
\text { Concentration }(\boldsymbol{\mu g} / \mathbf{m L})\end{array}$ & $\begin{array}{c}\text { Assay Value }(\mathbf{m e a n} \pm \\
\mathbf{S D})(\boldsymbol{\mu g} / \mathbf{m L})\end{array}$ & $\begin{array}{c}\text { Coefficient of } \\
\text { Variation } \mathbf{( \% )}\end{array}$ & $\begin{array}{c}\text { Accuracy } \\
\mathbf{( \% )}\end{array}$ \\
\hline adenosine & 42.00 & $41.65 \pm 0.27$ & 0.65 & 99.17 \\
2'-deoxyadenosine & 40.00 & $39.77 \pm 0.62$ & 1.56 & 99.43 \\
cordycepin & 42.00 & $42.39 \pm 0.54$ & 1.27 & 100.93 \\
Inter-day ${ }^{2}$ & & & & \\
uracil & 40.00 & $39.71 \pm 0.48$ & 1.21 & 99.28 \\
uridine & 40.00 & $39.55 \pm 0.73$ & 1.85 & 98.88 \\
2'-deoxyuridine & 70.00 & $70.82 \pm 1.25$ & 1.77 & 101.17 \\
inosine & 38.00 & $37.62 \pm 0.74$ & 1.97 & 99.00 \\
guanosine & 40.00 & $40.61 \pm 0.93$ & 2.29 & 101.53 \\
adenine & 40.00 & $39.63 \pm 0.68$ & 1.72 & 99.08 \\
thymidine & 86.00 & $85.17 \pm 1.27$ & 1.49 & 99.03 \\
adenosine & 42.00 & $41.57 \pm 0.45$ & 1.08 & 98.98 \\
2'-deoxyadenosine & 40.00 & $39.62 \pm 0.84$ & 2.12 & 99.05 \\
cordycepin & 42.00 & $42.49 \pm 0.69$ & 1.62 & 101.17 \\
\hline
\end{tabular}

${ }^{\mathrm{d}}$ The sample was analyzed five times within one day (intra-day) and over two consecutive days (inter-day).

\subsubsection{Extraction Recoveries}

Recoveries and reproducibility of the proposed methods for target compounds were calculated using the $C$. cicadae (population CCLTL) mixture sample as a representative. The extraction recovery was performed by adding a known amount of individual standards into a $0.50 \mathrm{~g}$ of C. cicadae sample. Three replicates were performed for the test. The mixture was extracted and analyzed using the method mentioned above. Table 8 shows the recoveries of 10 nucleosides.

Table 8. Recoveries for the assay of 10 nucleosides in C. cicadae $(\mathrm{n}=3)$.

\begin{tabular}{cccccc}
\hline Analyte & $\begin{array}{c}\text { Original } \\
(\boldsymbol{\mu g})\end{array}$ & $\begin{array}{c}\text { Spiked Amount } \\
(\boldsymbol{\mu g})\end{array}$ & $\begin{array}{c}\text { Found }^{\mathrm{e}}(\mathbf{m e a n} \pm \mathbf{S D}) \\
(\boldsymbol{\mu g})\end{array}$ & $\begin{array}{c}\text { Recovery } \\
\mathbf{( \% )}\end{array}$ & $\begin{array}{c}\text { Coefficient of } \\
\text { Variation }(\mathbf{\%})\end{array}$ \\
\hline uracil & 145.35 & 140.00 & $282.08 \pm 4.89$ & 98.85 & 1.73 \\
uridine & 714.12 & 700.00 & $1398.28 \pm 11.60$ & 98.88 & 0.83 \\
2'-deoxyuridine & 63.85 & 60.00 & $126.87 \pm 2.36$ & 102.44 & 1.86 \\
inosine & 140.62 & 140.00 & $276.66 \pm 2.19$ & 98.59 & 0.79 \\
guanosine & 415.96 & 400.00 & $806.39 \pm 3.99$ & 98.83 & 0.50 \\
thymidine & 39.61 & 40.00 & $80.55 \pm 1.28$ & 101.18 & 1.59 \\
adenine & 31.96 & 30.00 & $61.05 \pm 0.70$ & 98.53 & 1.14 \\
adenosine & 375.96 & 370.00 & $736.62 \pm 4.81$ & 98.75 & 0.65 \\
2'-deoxyadenosine & 25.31 & 30.00 & $54.20 \pm 0.76$ & 97.99 & 1.40 \\
cordycepin & $-\mathrm{g}$ & 150.00 & $152.12 \pm 0.78$ & 101.41 & 0.51 \\
\hline
\end{tabular}

${ }^{\mathrm{e}}$ The data were present as an average of three determinations; ${ }^{\mathrm{f}}$ Recovery $(\%)=100 \times(($ amount found - original amount)/amount spiked); ${ }^{\mathrm{g}}$ Not detected.

\subsection{Statistical Analysis}

The data were statistically analyzed using the Statistical Analysis System (SAS) 8.1 software. 


\section{Conclusions}

Simple and convenient HPLC methods for the determination of the content of nucleosides in $C$. cicadae populations were described. The method might be used for fast determination of the nucleosides in Cordyceps materials.

Chemical constituents of natural crude drugs, including C. cicadae occurring in Nature, are affected by location, geography, climate and microenvironment. The variance of nucleosides was large in natural $C$. cicadae, and might be derived from genetic differences. The genetic differentiation of C. cicadae populations by DALP and EST-SSR will be discussed in future papers.

The use of $C$. cicadae as a Traditional Chinese Medicine and tonic food has been appreciated for more than 1,500 years, and it has been used as a substitute for $O$. sinensis. The content and distribution of nucleosides in $C$. cicadae were similar to those in $O$. sinensis, and the medicinal effectiveness of $C$. cicadae was also similar to that of $O$. sinensis. Furthermore, the habitat demands of $C$. cicadae are less strict than those of $O$. sinensis, and its resource distribution and reserves were much larger than those of $O$. sinensis. The price of $C$. cicadae was about 2,000 yuan per kilogram in 2013, which was $1 / 100$ of that of $O$. sinensis. It was suggested that $C$. cicadae should be used as substitute for $O$. sinensis.

\section{Acknowledgments}

This work was supported by the Specialized Research Fund for the Doctoral Program of Higher Education (20125301110001) and Yunnan Natural Science Foundation of China (2008CC019).

\section{Author Contributions}

Wen-Bo Zeng, Hong Yu and Feng Ge designed research; Wen-Bo Zeng, Jun-Yuan Yang, Zi-Hong Chen, Yuan-Bing Wang and Yong-Dong Dai performed experiments and analyzed the data; Wen-Bo Zeng, Hong Yu, Yuan-Bing Wang and Yong-Dong Dai collected the Cordyceps materials, Wen-Bo Zeng, Hong Yu and Alison Adams wrote the paper. All authors read and approved the manuscript.

\section{Conflicts of Interest}

The authors declare no conflict of interest.

\section{References}

1. Luangsa-Ard, J.J.; Hywel-Jones, N.L.; Manoch, L.; Samson, R.A. On the relationships of Paecilomyces sect. Isarioidea species. Mycol. Res. 2005, 109, 581-589.

2. Li, B.L. Herbal textuals research on "Chan Hua". Chin. J. Med. Appl. Pharm. 1993, 10, 21-22.

3. Chen, Z.A.; Liu, G.Y.; Hu, S.Y. Study on cultivation of Paecilomyces cicadae and its pharmacological function. Acta Mycol. Sin. 1993, 12, 138-144.

4. Li, S.P.; Yang, F.Q.; Tsim, K.W.K. Quality control of Cordyceps sinensis, a valued traditional Chinese medicine. J. Pharm. Biomed. 2006, 41, 1571-1584.

5. Sung, G.H.; Hywel-Jones, N.L.; Sung, J.M.; Luangsa-Ard, J.J.; Shrestha, B.; Spatafora, J.W. Phylogenetic classification of Cordyceps and the clavicipitaceous fungi. Stud. Mycol. 2007, 57, 5-59. 
6. Ukai, S.; Kiho, T.; Hara, C.; Morita, M.; Goto, A.; Imaizumi, N.; Hasegawa, Y. Polysaccharides in Fungi. XIII. Antitumor activity of various polysaccharides isolated from Dictyophora indusiata, Ganoderma japonicum, Cordyceps cicadae, Auricularia auricula-judae and Auricularia species. Chem. Pharm. Bull. 1983, 31, 741-744.

7. Weng, S.C.; Chou, C.J.; Lin, L.C.; Tsai, W.J.; Kuo, Y.C. Immunomodulatory functions of extracts from the Chinese medicinal fungus Cordyceps cicadae. J. Ethnopharmacol. 2002, 83, 79-85.

8. Liu, G.Y.; Hu, S.Y. Comparison of sedative and analgesic effects between Cordyceps cicadae and its cultured product. Chin. J. Med. Appl. Pharm. 1991, 8, 4-8.

9. Zhu, R.; Chen, Y.P.; Deng, Y.Y.; Zheng, R.; Zhong, Y.F.; Wang, L.; Du, L.P. Cordyceps cicadae extracts ameliorate renal malfunction in a remnant kidney model. J. Zhejiang Univ. Sci. B 2011, $12,1024-1033$.

10. Wang, Y.; Zhao, X.J.; Tang, F.D. Primary exploring on pharmic effect of Cordyceps cicadae. Zhejiang J. Chin. Tradit. Med. 2001, 36, 219-220.

11. Bi, S.Z.; Liu, B.; Ying, J.Z.; Shao, L.P.; Huang, N.L.; Zhang, D.Z.; Xie, Z.X.; Zang, M.; Wei, R.Q. Edible Fungal Flora of China; China Forestry Publishing House: Shanghai, China, 1991; p. 13.

12. Liu, A.Y.; Li, Z.; Zhou, X.; Zhao, H.J.; Hu, H.Y.; Tan, A.J. Research and Application of Cordyceps cicadae Resources in China; Guizhou science and Technology Press: Guiyang, China, 2012; pp. 34-52.

13. Liu, A.Y.; Zhou, X.; Zhao, H.J.; Liang, Z.Q.; Tan, A.J.; Zheng, Q.Y. Biological diversity of Paecilomyces cicadae I. Morphological diversity of cicadae flower and Paecilomyces cicadae. Guizhou Agric. Sci. 2007, 35, 9-11.

14. Zhang, Y.J.; Liu, A.Y.; Liang, Z.Q. Formation and regeneration of protoplasts from Paecilomyces cicadae. Guizhou Agric. Sci. 1998, 26, 1-4.

15. Feng, L.C. Study on TianMa Hill Cordyceps cicadae. J. Shanghai Inst. Technol. 2002, 2, 125-127.

16. Chen, D.Q.; Ding, Z.S.; Lin, A.M.; Pan, P.L.; Chen, Y.T. Isolation and fermentation culture of fungi from Cordyceps cicadae. J. Chin. Med. Mater. 2006, 29, 99-101.

17. Wang, Q.; Liu, Z.Y. Advances in studies on medicinal fungi Cordyceps cicadae. Chin. Tradit. Herb. Drugs 2004, 34, 469-471.

18. Kiho, T.; Ito, M.; Nagai, K.; Hara, C.; Ukai, S. Polysaccharides in fungi. X XII. a water soluble polysaccharide from the alkaline extract of the insect-body portion of Chan hua (fungus: Cordyceps cicadae). Chem. Pharm. Bull. 1988, 36, 3032-3037.

19. Kim, H.S.; Kim, J.Y.; Ryu, H.S.; Shin, B.R.; Kang, J.S.; Kim, H.M.; Kim, Y.O.; Hong, J.T.; Kim, Y.; Han, S.B. Phenotypic and functional maturation of dendritic cells induced by polysaccharide isolated from Paecilomyces cicadae. J. Med. Food. 2011, 14, 847-856.

20. Kiho, T.; Nagai, K.; Miyamoto, I.; Watanabe, T.; Ukai, S. Polysaccharides in fungi. XXV. Biological activities of two galactomannans from the insect-body portion of Chan hua (fungus: Cordyceps cicadae). Yakugaku Zasshi 1990, 110, 286-288.

21. Ge, F.; Xia, C.R.; LI, C.R.; Ding, T.; Shao, Y.; Fan, M.Z. Analysis of the chemical compositions of Paecilomyces cicadae fermented mycelia and Cordyceps cicadae fruit body. Mycosystema 2007, 26, 68-75. 
22. Osuchowski, M.F.; Johnson, V.J.; He, Q.R.; Sharma, R.P. Myriocin, a serine palmitoyltransferase inhibitor, alters regional brain neurotransmitter levels without concurrent inhibition of the brain sphingolipid biosynthesis in mice. Toxicol. Lett. 2004, 147, 87-94.

23. Yu, J.W.; Xu, H.J.; Mo, Z.H.; Zhu, H.L.; Mao, X.B. Determination of myriocin in natural and cultured Cordyceps cicadae using 9-fluorenylmethyl chloroformate derivatization and high-performance liquid chromatography with UV-detection. Anal. Sci. 2009, 25, 855-859.

24. Kuo, Y.C.; Weng, S.C.; Chou, C.J.; Chang, T.T.; Tsai, W.J. Activation and proliferation signals in primary human $\mathrm{T}$ lymphocytes inhibited by ergosterol peroxide isolated from Cordyceps cicadae. Br. J. Pharmacol. 2003, 140, 895-906.

25. Cunningham, K.G.; Manson, W.; Spring, F.S.; Hutchinson, S.A. Cordycepin, a metabolic product isolated from cultures of Cordyceps militaris (Linn.) Link. Nature 1950, 166, 949.

26. Fan, H.; Li, S.P.; Xiang, J.J.; Lai, C.M.; Yang, F.Q.; Gao, J.L.; Wang, Y.T. Qualitative and quantitative determination of nucleosides, bases and their analogues in natural and cultured Cordyceps by pressurized liquid extraction and high performance liquid chromatography-electrospray ionization tandem mass spectrometry (HPLC-ESI-MS/MS). Anal. Chim. Acta 2006, 567, 218-228.

27. Ikeda, R.; Nishimura, M.; Sun, Y.; Wada, M.; Nakashima, K. Simple HPLC-UV determination of nucleosides and its application to the authentication of Cordyceps and its allies. Biomed. Chromatogr. 2008, 22, 630-636.

28. Coradetti, R.; Conte, G.L.; Moroni, F.; Passani, M.B.; Pepeu, G. Adenosine decreases aspartate and glutamate release from rat hippocampal slices. Eur. J. Pharmacol. 1984, 104, 19-26.

29. Schmidt, C.; Bellingham, M.C.; Richter, D.W. Adenosinergic modulation of respiratory neurones and hypoxic responses in the anaesthetized cat. J. Physiol. 1995, 483, 769-781.

30. The Pharmacopoeia Commission of PRC. Pharmacopoeia of the People's Republic of China, 9th ed.; Chemical Industry Publishing House: Beijing, China, 2010; pp. 106.

31. Benowitz, L.I.; Goldberg, D.E.; Irwin, N. Inosine stimulates axon growth in vitro and in the adult CNS. Prog. Brain Res. 2002, 137, 389-399.

32. Zhou, X.X.; Meyer, C.U.; Schmidtke, P.; Zepp, F. Effect of cordycepin on interleukin-10 production of human peripheral blood mononuclear cells. Eur. J. Pharmacol. 2002, 453, 309-317.

33. Ahn, Y.J.; Park, S.J.; Lee, S.G.; Shin, S.C.; Choi, D.H. Cordycepin: Selective growth inhibitor derived from liquid culture of Cordyceps militaris against Clostridium spp. J. Agric. Food Chem. 2000, 48, 2744-2748.

34. Kodama, E.N.; McCaffrey, R.P.; Yusa, K.; Mitsuya, H. Antileukemic activity and mechanism of action of cordycepin against terminal deoxynucleotidal transferase-positive $(\mathrm{TdT}+)$ leukemic cells. Biochem. Pharmacol. 2000, 59, 273-281.

35. Li, S.P.; Li, P.; Lai, C.M.; Gong, Y.X.; Kan, K.K.W.; Dong, T.T.X.; Tsim, K.W.K.; Wang, Y.T. Simultaneous determination of ergosterol, nucleosides and their bases from natural and cultured Cordyceps by pressurised liquid extraction and high-performance liquid chromatography. J. Chromatogr. A 2004, 1036, 239-243.

36. Guo, F.Q.; Li, A.; Huang, L.F.; Liang, Y.Z.; Chen, B.M. Identification and determination of nucleosides in Cordyceps sinensis and its substitutes by high performance liquid chromatography with mass spectrometric detection. J. Pharm. Biomed. 2006, 40, 623-630. 
37. Yang, F.Q.; Li, S.P. Effects of sample preparation methods on the quantification of nucleosides in natural and cultured Cordyceps. J. Pharm. Biomed. 2008, 48, 231-235.

38. Uauy, R.; Stringel, G.; Thomas, R.; Quan, R. Effect of dietary nucleosides on growth and maturation of the developing Gut in the Rat. J. Pediatr. Gastroenterol. Nutr. 1994, 10, 497-503.

39. Carver, J.D.; Walker, W.A. The role of nucleotides in human nutrition. J. Nutr. Biochem. 1995, 6, $58-72$.

40. Wang, S.; Yang, F.Q.; Feng, K.; Li, D.Q.; Zhao, J.; Li, S.P. Simultaneous determination of nucleosides, myriocin, and carbohydrates in Cordyceps by HPLC coupled with diode array detection and evaporative light scattering detection. J. Sep. Sci. 2009, 32, 4069-4076.

41. Wang, Z.B.; Li, N.; Wang, M.; Wang, Y.; Du, L.; Ji, X.F.; Yu, A.M.; Zhang, H.Q.; Qiu, F.P. Simultaneous determination of nucleosides and their bases in Cordyceps sinensis and its substitutes by matrix solid-phase dispersion extraction and HPLC. J. Sep. Sci. 2013, 36, 2348-2357.

42. Yang, F.Q.; Li, D.Q.; Feng, K.; Hu, D.J.; Li, S.P. Determination of nucleotides, nucleosides and their transformation products in Cordyceps by ion-pairing reversed-phase liquid chromatography-mass spectrometry. J. Chromatogr. A 2010, 1217, 5501-5510.

43. Huang, L.F.; Liang, Y.Z.; Guo, F.Q.; Zhou, Z.F.; Cheng, B.M. Simultaneous separation and determination of active components in Cordyceps sinensis and Cordyceps militaris by LC/ESI-MS. J. Pharm. Biomed. 2003, 33, 1155-1162.

44. Yang, F.Q.; Ge, L.Y.; Yong, J.W.H.; Tan, S.N.; Li, S.P. Determination of nucleosides and nucleobases in different species of Cordyceps by capillary electrophoresis-mass spectrometry. J. Pharm. Biomed. 2009, 50, 307-314.

45. Gong, Y.X.; Li, S.P.; Li, P.; Liu, J.J.; Wang, Y.T. Simultaneous determination of six main nucleosides and bases in natural and cultured Cordyceps by capillary electrophoresis. J. Chromatogr. A 2004, 1055, 215-221.

46. Yang, F.Q.; Li, S.P.; Li, P.; Wang, Y.T. Optimization of CEC for simultaneous determination of eleven nucleosides and nucleobases in Cordyceps using central composite design. Electrophoresis 2007, 28, 1681-1688.

47. Yang, F.Q.; Guan, J.; Li, S.P. Fast simultaneous determination of 14 nucleosides and nucleobases in cultured Cordyceps using ultra-performance liquid chromatography. Talanta 2007, 73, 269-273.

48. Ling, J.Y.; Sun, Y.J.; Zhang, H.; Lv, P.; Zhang, C.K. Measurement of cordycepin and adenosine in stroma of Cordyceps sp. by capillary zone electrophoresis (CZE). J. Biosci. Bioeng. 2002, 94, 371-374.

49. Li, S.P.; Li, P.; Ji, H.; Zhang, P.; Dong, T.T.X.; Tsim, K.W.K. The contents and their change of nucleosides from natural Cordyceps sinensis and cultured Cordyceps mycelia. Acta Pharm. Sin. 2001, 36, 436-439.

50. Rao, Y.K.; Chou, C.H.; Tzeng, Y.M. A simple and rapid method for identification and determination of cordycepin in Cordyceps militaris by capillary electrophoresis. Anal. Chim. Acta 2006, 566, 253-258.

51. Yuan, J.P.; Zhao, S.Y.; Wang, J.H.; Kuang, H.C.; Liu, X. Distribution of nucleosides and nucleobases in Edible Fungi. J. Agric. Food Chem. 2008, 56, 809-815.

52. Gu, Y.X.; Wang, Z.S.; Li, S.X.; Yuan, Q.S. Effect of multiple factors on accumulation of nucleosides and bases in Cordyceps militaris. Food Chem. 2007, 102, 1304-1309. 
53. Yu, L.; Zhao, J.; Li, S.P.; Fan, H.; Hong, M.; Wang, Y.T.; Zhu, Q. Quality evaluation of Cordyceps through simultaneous determination of eleven nucleosides and bases by RP-HPLC. J. Sep. Sci. 2006, 29, 953-958.

54. Li, S.P.; Su, Z.R.; Dong, T.T.X.; Tsim, K.W.K. The fruiting body and its caterpillar host of Cordyceps sinensis show close resemblance in main constituents and anti-oxidation activity. Phytomedicine 2002, 9, 319-324.

55. Xie, J.W.; Huang, L.F.; Hu, W.; He, Y.B.; Wong, K.P. Analysis of the main nucleosides in Cordyceps sinensis by LC/ESI-MS. Molecules 2010, 15, 305-314.

56. Hsu, T.H.; Shiao, L.H.; Hsieh, C.; Chang, D.M. A comparison of the chemical composition and bioactive ingredients of the Chinese medicinal mushroom DongChongXiaCao, its counterfeit and mimic, and fermented mycelium of Cordyceps sinensis. Food Chem. 2002, 78, 463-469.

57. Zhu, J.S.; Halpern, G.M.; Jones, K. The scientific rediscovery of an ancient Chinese herbal medicine: Cordyceps sinensis: Part I. J. Altern. Complement. Med. 1998, 4, 289-303.

58. Zhu, J.S.; Halpern, G.M.; Jones, K. The scientific rediscovery of a precious ancient Chinese herbal regimen: Cordyceps sinensis: Part II. J. Altern. Complement. Med. 1998, 4, 429-457.

59. Li, S.P.; Li, P.; Dong, T.T.X.; Tsim, K.W.K. Determination of nucleosides in natural Cordyceps sinensis and cultured Cordyceps mycelia by capillary electrophoresis. Electrophoresis 2001, 22, 144-150.

60. Jiang, H.; Liu, K.; Meng, S.; Chu, Z.Y. Chemical constituents of the dry sorophore of Cordyceps militaris. Acta Pharm. Sin. 2000, 35, 663-668.

61. Yu, H.M.; Wang, B.S.; Huang, S.C.; Duh, P.D. Comparison of protective effects between cultured Cordyceps militaris and natural Cordyceps sinensis on oxidative damage. J. Agric. Food Chem. 2006, 54, 3132-3138.

Sample Availability: Not available.

(C) 2014 by the authors; licensee MDPI, Basel, Switzerland. This article is an open access article distributed under the terms and conditions of the Creative Commons Attribution license (http://creativecommons.org/licenses/by/3.0/). 